\title{
Remarks on simple interpolation between Jordanian twists
}

This content has been downloaded from IOPscience. Please scroll down to see the full text.

Download details:

IP Address: 161.23.96.94

This content was downloaded on 25/05/2017 at 10:01

Manuscript version: Accepted Manuscript

Meljanac et al

To cite this article before publication: Meljanac et al, 2017, J. Phys. A: Math. Theor, at press: https://doi.org/10.1088/1751-8121/aa72d7

This Accepted Manuscript is: (c) 2017 IOP Publishing Ltd

During the embargo period (the 12 month period from the publication of the Version of Record of this article), the Accepted Manuscript is fully protected by copyright and cannot be reused or reposted elsewhere.

As the Version of Record of this article is going to be / has been published on a subscription basis, this Accepted Manuscript is available for reuse under a CC BY-NC-ND 3.0 licence after the 12 month embargo period.

After the embargo period, everyone is permitted to copy and redistribute this article for non-commercial purposes only, provided that they adhere to all the terms of the licence https://creativecommons.org/licences/by-nc-nd/3.0

Although reasonable endeavours have been taken to obtain all necessary permissions from third parties to include their copyrighted content within this article, their full citation and copyright line may not be present in this Accepted Manuscript version. Before using any content from this article, please refer to the Version of Record on IOPscience once published for full citation and copyright details, as permission will likely be required. All third party content is fully copyright protected, unless specifically stated otherwise in the figure caption in the Version of Record.

When available, you can view the Version of Record for this article at:

http://iopscience.iop.org/article/10.1088/1751-8121/aa72d7 


\title{
Remarks on simple interpolation between Jordanian twists
}

\author{
Stjepan Meljanac ${ }^{* 1}$, Daniel Meljanac ${ }^{\dagger 1}$, Anna Pachoł ${ }^{\ddagger 2}$ and Danijel Pikutić ${ }^{\S 1}$
}

${ }^{1}$ Division of Theoretical Physics, Ruđer Bošković Institute, Bijenička c.54, HR-10002 Zagreb, Croatia

${ }^{2}$ Queen Mary, University of London, School of Mathematics, Mile End Rd., London E1 4NS, UK.

December 29, 2016

\begin{abstract}
In this paper, we propose a simple generalization of the locally $r$-symmetric Jordanian twist, resulting in the one-parameter family of Jordanian twists. All the proposed twists differ by the coboundary twists and produce the same Jordanian deformation of the corresponding Lie algebra. They all provide the $\kappa$-Minkowski spacetime commutation relations. Constructions from noncommutative coordinates to the star product and coproduct, and from the star product to the coproduct and the twist are presented. The corresponding twist in the Hopf algebroid approach is given. Our results are presented symbolically by a diagram relating all of the possible constructions.
\end{abstract}

\section{Introduction}

In the Hopf algebras framework it is known that the given Hopf algebra $\mathcal{H}(\mu, \Delta, \epsilon, S)$ can be deformed by using the (Drinfeld) twist $\mathcal{F} \in \mathcal{H} \otimes \mathcal{H}[1$ which allows to deform the coproduct and an antipode map in such a way that the compatibility conditions are still satisfied and one gets a new Hopf algebra with deformed maps. One special example of such twist is the so-called Jordanian twist $\mathcal{F}_{0}=\exp (\ln (1+\alpha E) \otimes H)$ with $\alpha$ as the deformation parameter 1 . It was firstly constructed by O. Ogievetsky in [2] while studying the moduli space of Hopf structures on the Borel subalgebra of $s l(2) \ni\{H, E \mid[H, E]=E\}$. This twist provides the simplest example of the triangular deformation of $s l(2)$ algebra with the quantum R-matrix given by $R=\tilde{\mathcal{F}} \mathcal{F}^{-1}=1 \otimes 1+r+\mathcal{O}\left(\alpha^{2}\right)$, where $\tilde{\mathcal{F}}=\tau \circ \mathcal{F}$ is the flipped twist (with the flip map: $\tau: c \otimes d \rightarrow d \otimes c$ ) and $r$ is denoting the classical r-matrix.

*meljanac@irb.hr

†Daniel.Meljanac@irb.hr

${ }_{\ddagger}^{\ddagger}$ a.pachol@qmul.ac.uk

$\S$ dpikutic@irb.hr

${ }^{1}$ To distinguish different Jordanian twists we introduce a notation with sub-indices 0,1 , or $1 / 2$ which will become clear in section 2 .

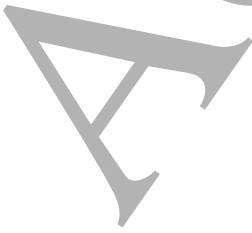


One can notice that $\mathcal{F}_{0}$ is not r-symmetric [3] in the sense that the term of the expansion in the deformation parameter $\alpha$, at the first order, is not given by the full classical r-matrix $r$, i.e. is not of the form: $\mathcal{F}=1 \otimes 1+\frac{1}{2} r+\mathcal{O}\left(\alpha^{2}\right)$. However it can be symmetrized by the so called coboundary twist $\mathcal{F}_{\omega}=\omega^{-1} \otimes \omega^{-1} \Delta(\omega)$ as:

$$
\mathcal{F}_{r}^{(\omega)}:=\omega^{-1} \otimes \omega^{-1} \mathcal{F}_{0} \Delta(\omega)
$$

where $\omega=\sqrt{\mu\left[(1 \otimes S) \mathcal{F}_{0}\right]}$. The change of the twist by the coboundary twist $\mathcal{F}_{\omega}$ does not provide a new Hopf algebra. It provides a new presentation in the co-algebraic sector only, i.e. new form of the coproduct $\tilde{\Delta}=\mathcal{F}_{\omega} \Delta \mathcal{F}_{\omega}^{-1}$.

One of the symmetrized versions (i.e. "locally r-symmetric") of the Jordanian twist $\tilde{\mathcal{F}}=\tau \circ \mathcal{F}_{0}$ known in the literature is due to V. N. Tolstoy [3] which appeared in the context of studying quantum deformations of relativistic symmetries. The locally r-symmetric version of the Jordanian twist calculated therein has the form:

$$
\mathcal{F}_{1 / 2}=e^{\frac{\alpha}{2}(H E \otimes 1+1 \otimes H E)} e^{H \otimes \ln (1+\alpha E)} e^{-\frac{\alpha}{2}(H E \otimes 1+H \otimes E+E \otimes H+1 \otimes H E)}
$$

Here $\omega=\exp \left(\frac{1}{2} \alpha H E\right)$ was used to construct the coboundary twist. There exists another version of symmetrized $\mathcal{F}_{0}$ and it is due to Giaquinto and Zhang 4 , but it/will not be the object of our study here.

The Jordanian twist investigated, firstly in [2] and then in [3], reappeared in the context of the so-called $\kappa$-Minkowski noncommutative spacetime [5, 6, 7], which is an algebra of coordinate functions equipped in the noncommutative star-product leading to the following commutation relations $\left[\hat{x}^{\mu}, \hat{x}^{\nu}\right]=\frac{i}{\kappa}\left(v^{\mu} \hat{x}^{\nu}-v^{\nu} \hat{x}^{\mu}\right)$ where the deformation parameter is $\frac{1}{\kappa}$ and $v^{\mu}$ is the vector on Minkowski spacetime $\mathcal{M}_{1, n-1}$ in $n$-dimensions such that $v^{2} \in\{-1,0,1\}$. In physical applications $\kappa$ is usually interpreted as the Planck mass or Quantum Gravity scale. The natural quantum symmetry of this noncommutative spacetime is the $\kappa$-Poincaré quantum group [8] and it constitutes one of the examples of deformed relativistic spacetime symmetries. In this paper we are interested in the relation with the symmetry of the $\kappa$-Minkowski spacetime therefore we shall work with the generators of relativistic symmetries: dilatation $D$ and momenta $p_{\alpha}$ (instead of the generators $H$ and $E$ of the $s l(2)$ algebra) satisfying the same commutation relation, i.e. $\left[p_{\alpha}, D\right]=p_{\alpha}$ 2. The dilatation generator $D$ is included in the minimal extension of the relativistic spacetime symmetry, the so-called Poincaré-Weyl symmetry: $\left\{M_{\mu \nu}, p_{\mu}, D\right\}$ as well as in the conformal algebra $\left\{M_{\mu \nu}, p_{\mu}, D, K_{\mu}\right\}$, therefore the Jordanian twists have the support in both of these algebras and can be used in their deformations [5], [9], respectively. However for the presentation of our results it is enough to consider the Lie algebra $g$ generated only by the dilatation and momenta operators satisfying $\left[p_{\alpha}, D\right]=p_{\alpha}$ and $\left[p_{\mu}, p_{\nu}\right]=0$. Recently Jordanian twists also have been considered in application to gravitational theory. For example, in [11, the Jordanian twist was used to construct the noncommutative differential calculus providing metrics as the solutions of non-vacuum Einstein equations, including cosmological constant or spatial curvature cases. Additionally, it can be shown [10] that the star product, reproducing the $\kappa$ - Minkowski Lie algebra, obtained by suitably reducing the so-called Wick-Voros star product, is in fact the star product from the Jordanian twist $\mathcal{F}_{0}$. Jordanian deformations/also have become popular in the context of applications in AdS/CFT correspondence $[12$.

In this paper we are interested in the generalization of the symmetrized version of Jordanian twist $\mathcal{F}_{1 / 2}$ from [3]. We introduce the real parameter characterizing a whole family of Jordanian twists

2 The correspondence with the generators of $\operatorname{sl}(2)$ algebra is $H \rightarrow-D$ and $E \rightarrow p_{\alpha}$. 
interpolating between the original Jordanian twist $\mathcal{F}_{0}=\exp \left(-\ln \left(1-a^{\alpha} p_{\alpha}\right) \otimes D\right)$ and Jordanian twist $\mathcal{F}_{1}=\left.\tau \circ \mathcal{F}_{0}\right|_{-a^{\alpha}}=\exp \left(-D \otimes \ln \left(1+a^{\alpha} p_{\alpha}\right)\right)$, where $a^{\alpha}=\frac{1}{\kappa} v^{\alpha}$ (in section 2). They both lead to the $\kappa$-Minkowski spacetime commutation relations. The aim of this paper is to present different methods to obtain the same family of twists 3 developed in the framework of noncommutative spacetimes.

In section 2, a generalization of the r-symmetric twist from [3] is given. Corresponding deformed Hopf algebra symmetry, star products and differential realizations for noncommutative coordinates are presented. This family of Jordanian twists is also constructed as one exponential formula. However, in general, the twist might not always be known (e.g. in examples coming from deformation quantization framework).

In section 3, we provide an example of a method used to construct the twist. Starting from realizations of noncommutative coordinates, obtained in section 2, we construct the corresponding star product and coproduct. Also, from the star product and the coproduct, presented in section 2 (and coinciding with those in section 3), inverses of corresponding twists are obtained. They differ from the ones presented in section 2 by the right ideal, but indeed give the same deformed Hopf algebra, star product and realization of noncommutative coordinates. At the end of section 3, twists in Hopf algebroid approach, in terms of momenta and noncommutative coordinates, are given, showing that the used techniques appear within this more general framework. In section 4 , concluding remarks are presented.

\subsection{Notation and formalism}

Here we will present the notation and recall some standard formulas related to the twist formalism which will be necessary for the following sections of the paper.

The Lie algebra $g$ generated by the dilatation and momenta operators is defined by the commutation relations:

$$
\left[p_{\mu}, D\right]=p_{\mu}, \quad\left[p_{\mu}, p_{\nu}\right]=0 .
$$

The differential representation of the generators is the following: $D=x^{\alpha} \partial_{\alpha}=x \cdot \partial$ and $p_{\mu}=-i \partial_{\mu}$. We will also use the short notation for the momenta $p_{\mu}$ contracted ${ }^{4}$ with the vector $a^{\nu}$ as $A=$ $i a^{\nu} \partial_{\nu}=-a^{\nu} p_{\nu}=-a \cdot p$. We do not specify signature of the metric, therefore the metric may be of any signature.

The generator $D$ can be also rewritten in Heisenberg realization as $D=i x^{\alpha} p_{\alpha}=i x \cdot p$, in terms of Heisenberg algebra $H$, i.e.:

$$
\left[x^{\mu}, x^{\nu}\right]=0, \quad\left[p_{\mu}, x^{\nu}\right]=-i \delta_{\mu}^{\nu}, \quad\left[p_{\mu}, p_{\nu}\right]=0 .
$$

The deformation of the Hopf algebra $U(g)\left(\mu, \Delta_{0}, \epsilon, S_{0}\right)$ of the universal enveloping algebra of $g=\left\{p_{\alpha}, D:\left[p_{\alpha}, D\right]=p_{\alpha},\left[p_{\mu}, p_{\nu}\right]=0\right\}$ given by the twist element $\mathcal{F} \in U(g)\left[\left[\frac{1}{\kappa}\right]\right] \otimes U(g)\left[\left[\frac{1}{\kappa}\right]\right]$ into $U^{\mathcal{F}}(g)(\mu, \Delta, \epsilon, S)$ is provided by the deformation of the coproduct and antipode maps as follows: $\Delta h=\mathcal{F} \Delta_{0} h \mathcal{F}^{-1}, S(h)=[\mu((1 \otimes S) \mathcal{F})] S_{0}(h)\left[\mu\left((S \otimes 1) \mathcal{F}^{-1}\right)\right]$, where $h \in g$.

The algebra of coordinates $\mathcal{A}$ with multiplication map $m: \mathcal{A} \otimes \mathcal{A} \rightarrow \mathcal{A}$, i.e. the spacetime algebra is the Hopf module algebra with the action $U(g) \otimes \mathcal{A} \rightarrow \mathcal{A}$ of the Hopf algebra $U(g)$ on the module algebra $\mathcal{A} \ni f, g$ such that $h \triangleright(m(f \otimes g))=m[\Delta h(\triangleright \otimes \triangleright)(f \otimes g)]$, where $h \in g$ and the

${ }^{3}$ up to the right ideal

${ }^{4}$ Note that here we are using the relativistic notation: $a^{\nu} b_{\nu}=a \cdot b$ (summation over $\nu$ index is assumed).

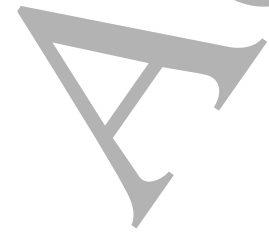


action $\triangleright$ is defined by

$$
p_{\mu} \triangleright f(x)=-i \partial_{\mu} \triangleright f(x)=-i \frac{\partial f(x)}{\partial x^{\mu}} \text { and } D \triangleright f(x)=x^{\alpha} \frac{\partial f(x)}{\partial x^{\alpha}}
$$

and the module acts on itself as $x^{\mu} \triangleright f(x)=x^{\mu} f(x)$.

The algebra of functions $\mathcal{A}$ becomes noncommutative during the twist deformation once the usual multiplication is replaced by the star-product between the functions i.e.:

$$
f \star g=m_{\star}(f \otimes g)=m\left[\mathcal{F}^{-1}(\triangleright \otimes \triangleright)(f \otimes g)\right]
$$

for $f, g \in \mathcal{A}$. The star product is associative (due to the fact that the twist $\mathcal{F}$ satisfies cocycle condition).

\section{Simple one-parameter family of Jordanian twists}

Let us introduce the following one-parameter family of twists $\mathcal{F}_{u} \in U(g)\left[\left[\frac{1}{\kappa}\right]\right] \otimes U(g)\left[\left[\frac{1}{\kappa}\right]\right]$ :

$$
\mathcal{F}_{u}=\exp (-u(D A \otimes 1+1 \otimes D A)) \exp (-\ln (1+A) \otimes D) \exp \left(\Delta_{0}(u D A)\right), \quad u \in \mathbb{R},
$$

The twists $\mathcal{F}_{u}$ can be obtained from $\mathcal{F}_{0}$ by the transformation with the coboundary twist with the element $\omega=e^{u D A}$. Note that the deformation parameter $1 / \kappa$ is included in $A$ for the purpose of simplified notation. The twists $\mathcal{F}_{u}$ are Drinfeld twists [1] $\forall u \in \mathbb{R}$, they satisfy normalization and cocycle condition ${ }^{5}$ and generalize the construction by V. N. Tolstoy [3], (cf. (1]). The correspondence of this family of the Jordanian twists $\left(\sqrt[5]{3}\right.$ and the r-symmetric twist $\mathcal{F}_{1 / 2}$ is given by taking $u=\frac{1}{2}$ in (5). For $u=0$, twist (5) simplifies to $\mathcal{F}_{0}=\exp (-\ln (1+A) \otimes D)$ and for $u=1$, simplifies to the twist $\mathcal{F}_{1}=\left.\tau \circ \mathcal{F}_{0}\right|_{-a}=\left.\tau \mathcal{F}_{0}\right|_{-a} \tau=\exp (-D \otimes \ln (1-A))$.

The inverse of the above family of twists is

$$
\mathcal{F}_{u}^{-1}=\exp \left(-\Delta_{0}(u D A)\right) \exp (\ln (1+A) \otimes D) \exp (u(D A \otimes 1+1 \otimes D A)), \quad u \in \mathbb{R}
$$

Now we can use the standard formulae (from section 1.1) for describing the deformation of the Hopf algebra maps of $U^{\mathcal{F}}(g)(\mu, \Delta, \epsilon, S)$. The coproducts, star products and realizations in the rest of the paper depend on the parameter $u$, but for the sake of simplicity, the $u$-dependence will be omitted.

Deformed Hopf algebra

Coproducts $\Delta p_{\mu}$, corresponding to the above twist, for any parameter $u \in \mathbb{R}$ are

$$
\begin{aligned}
\Delta p_{\mu} & =\mathcal{F}_{u} \Delta_{0} p_{\mu} \mathcal{F}_{u}^{-1}=\frac{p_{\mu} \otimes(1-u A)+(1+(1-u) A) \otimes p_{\mu}}{1 \otimes 1+u(1-u) A \otimes A} \\
\Delta D & =\mathcal{F}_{u} \Delta_{0} D \mathcal{F}_{u}^{-1}=\left(D \otimes \frac{1}{1-u A}+\frac{1}{1+(1-u) A} \otimes D\right)(1 \otimes 1+u(1-u) A \otimes A)
\end{aligned}
$$

\footnotetext{
${ }^{5}$ It is based on the fact that the change of the twist by the coboundary twist $\mathcal{F}_{\omega}$ influences the change of the coproduct by $\tilde{\Delta}=\mathcal{F}_{\omega} \Delta \mathcal{F}_{\omega}^{-1}$ which is isomorphic to the coproduct $\Delta$. Also adding the real valued parameter $u$ will not influence the cocyclicity.
} 
The coproduct is coassociative. The corresponding antipode is given by

$$
\begin{aligned}
& S\left(p_{\mu}\right)=\frac{-p_{\mu}}{1+(1-2 u) A} \\
& S(D)=-D-(1-u) A D+u D A-\frac{u(1-u)^{2} A^{2}}{(1+(1-u) A)(1-u A)}
\end{aligned}
$$

The antipode is antihomomorphism. The corresponding counit is trivial, i.e. $\epsilon\left(p_{\mu}\right)=0, \epsilon(D)=0$ and $\epsilon(1)=1$.

Interesting special cases are $u=0$ (cf.[10]) and $u=1$ (cf.[5]).

- For $u=0$ we get:

$$
\begin{aligned}
\Delta p_{\mu} & =p_{\mu} \otimes 1+(1+A) \otimes p_{\mu} \\
\Delta D & =D \otimes 1+\frac{1}{1+A} \otimes D \\
S\left(p_{\mu}\right) & =\frac{-p_{\mu}}{1+A} \\
S(D) & =-(1+A) D
\end{aligned}
$$

- For $u=1$ :

$$
\begin{aligned}
\Delta p_{\mu} & =p_{\mu} \otimes(1-A)+1 \otimes p_{\mu} \\
\Delta D & =D \otimes \frac{1}{1-A}+1 \otimes D \\
S\left(p_{\mu}\right) & =\frac{-p_{\mu}}{1-A} \\
S(D) & =-D(1-A)
\end{aligned}
$$

\section{Star product}

The inverse of the twist $\mathcal{F}_{u}^{-1}$ also provides the star product between the functions, as indicated in equation (4) This star product is associative (due to the fact that the twist $\mathcal{F}_{u}(5)$ satisfies cocycle condition).

When we choose our functions to be exponential functions $e^{i k \cdot x}$ and $e^{i q \cdot x}$, then we define new function $\mathcal{D}_{\mu}(k, q)$ :

$$
e^{i k \cdot x} \star e^{i q \cdot x}=m\left[\mathcal{F}^{-1}(\triangleright \otimes \triangleright)\left(e^{i k \cdot x} \otimes e^{i q \cdot x}\right)\right]=e^{i \mathcal{D}_{\mu}(k, q) x^{\mu}},
$$

where $k, q \in \mathcal{M}_{1, n-1}-n$-dimensional Minkowski spacetime.

One can calculate explicitly that in the case of twist $\mathcal{F}_{u}(5)$ the function $\mathcal{D}_{\mu}(k, q)$ is given by

$$
\mathcal{D}_{\mu}(k, q)=\frac{k_{\mu}(1+u(a \cdot q))+(1-(1-u)(a \cdot k)) q_{\mu}}{1+u(1-u)(a \cdot k)(a \cdot q)} .
$$

Note that the function $\mathcal{D}_{\mu}(k, q)$ can be seen as rewriting the coproduct $\Delta p_{\mu}$ without using the tensor product notation (denoting left and right leg by $k$ and $q$ respectively). Therefore the relation between the coproduct $\Delta p_{\mu}$ and the function $\mathcal{D}_{\mu}(k, q)$ is given by

$$
\Delta p_{\mu}=\mathcal{D}_{\mu}(p \otimes 1,1 \otimes p),
$$


hence $\Delta p_{\mu}$ uniquely determines $\mathcal{D}_{\mu}(k, q)$. In the case $u=0, \mathcal{D}_{\mu}(k, q)=k_{\mu}+(1-a \cdot k) q_{\mu}$, while in the case $u=1, \mathcal{D}_{\mu}(k, q)=k_{\mu}(1+a \cdot q)+q_{\mu}$. Deformed addition of momenta is given by $(k \oplus q)_{\mu}=\mathcal{D}_{\mu}(k, q)$.

\section{Coordinates}

Noncommutative coordinates $\hat{x}^{\mu}$, corresponding to the twist $\mathcal{F}_{u}(\sqrt[5]{5}$, are given by

$$
\begin{aligned}
\hat{x}^{\mu}=m\left[\mathcal{F}_{u}^{-1}(\triangleright \otimes 1)\left(x_{\mu} \otimes 1\right)\right] & =x^{\mu}(1-u A)+i a^{\mu}(1-u) D(1-u A) \\
& =\left(x^{\mu}+i a^{\mu}(1-u) D\right)(1-u A)
\end{aligned}
$$

Alternatively, we notice that they can also be obtained from the coproducts 6

$$
\begin{aligned}
\hat{x}^{\mu} & =x^{\mu}+i x^{\alpha} m\left[\left(\Delta-\Delta_{0}\right) p_{\alpha}(\triangleright \otimes 1)\left(x^{\mu} \otimes 1\right)\right] \\
& =\left(x^{\mu}+i a^{\mu}(1-u) D\right)(1-u A) .
\end{aligned}
$$

The noncommutative coordinates $\hat{x}^{\mu}$ satisfy

$$
\begin{aligned}
& {\left[\hat{x}^{\mu}, \hat{x}^{\nu}\right]=i\left(a^{\mu} \hat{x}^{\nu}-a^{\nu} \hat{x}^{\mu}\right)} \\
& {\left[p_{\mu}, \hat{x}^{\nu}\right]=\left(-i \delta_{\mu}^{\nu}+i a^{\nu}(1-u) p_{\mu}\right)(1-u A) .}
\end{aligned}
$$

In the case $u=0, \hat{x}^{\mu}=x^{\mu}+i a^{\mu} D$, while in the case $u=1, \hat{x}^{\mu}=x^{\mu}(1-A)$.

Note that we can define another set of the noncommutative coordinates $\hat{y}^{\mu}$ coming from the flipped version of (5) as

$$
\begin{aligned}
\hat{y}^{\mu} & =m\left[\tilde{\mathcal{F}}_{u}^{-1}(\triangleright \otimes 1)\left(x^{\mu} \otimes 1\right)\right] \\
& =x^{\mu}+i x^{\alpha} m\left[\left(\tilde{\Delta}-\Delta_{0}\right) p_{\alpha}(\triangleright \otimes 1)\left(x^{\mu} \otimes 1\right)\right] \\
& =\left(x^{\mu}-i a^{\mu} u D\right)(1+(1-u) A),
\end{aligned}
$$

where $\tilde{\mathcal{F}}_{u}=\tau \circ \mathcal{F}_{u}=\tau \mathcal{F}_{u} \tau$ and $\tilde{\Delta}=\tau \circ \Delta=\tau \Delta \tau$. Generators $\hat{y}_{\mu}$ define a dual coordinate algebra in the sense:

$$
\left[\hat{y}^{\mu}, \hat{y}^{\nu}\right]=-i\left(a^{\mu} \hat{y}^{\nu}-a^{\nu} \hat{y}^{\mu}\right) \quad \text { and } \quad\left[\hat{x}^{\mu}, \hat{y}^{\nu}\right]=0
$$

i.e. they obey $\kappa$-Minkowski commutation relations with $a^{\mu} \rightarrow-a^{\mu}$. The commutation relation $\left[p_{\mu}, \hat{y}^{\nu}\right]$ follows from the realization $(25)$. In the case $u=0, \hat{y}^{\mu}=x^{\mu}(1+A)$, while in the case $u=1$, $\hat{y}^{\mu}=x^{\mu}-i a^{\mu} D$.

One exponent formula for a family of Jordanian twists (5)

Above family of twists $\mathcal{F}_{u}$ given by $(5)$ can be written as

where

$$
\mathcal{F}_{u}=\exp \left((D \otimes u A) \theta-\left.((1-u) A \otimes D) \tilde{\theta}\right|_{(-a)}\right)
$$

$$
\theta=\sum_{n=0}^{\infty} \sum_{\substack{k, l=0 \\ k+l=n}}^{n} c_{k, l} A^{k} \otimes A^{l} \times \begin{cases}(1-u)^{k} u^{l} & \text { for } u \in \mathbb{R} \backslash\{0,1\} \\ \delta_{l 0} & \text { for } u=0 \\ \delta_{k 0} & \text { for } u=1\end{cases}
$$

${ }^{6}$ Later on we introduce 43 from which this relation follows naturaly. 
where $c_{k, l} \in \mathbb{R}$ and $c_{0,0}=1$. The first three terms in the above expansion in $\frac{1}{\kappa}$ are:

$$
\begin{aligned}
\left(\ln \mathcal{F}_{u}\right)_{1} & =D \otimes u A-(1-u) A \otimes D \\
\left(\ln \mathcal{F}_{u}\right)_{2} & =\frac{1}{2}[D \otimes u A+(1-u) A \otimes D][1 \otimes u A+(1-u) A \otimes 1] \\
\left(\ln \mathcal{F}_{u}\right)_{3} & \left.=D \otimes u A\left[\frac{1}{3}\left(1 \otimes u^{2} A^{2}\right)+\frac{1}{6}(1-u) A \otimes u A-\frac{1}{6}(1-u)^{2} A^{2} \otimes 1\right]\right) \\
& -(1-u) A \otimes D\left[\frac{1}{3}(1-u)^{2} A^{2} \otimes 1+\frac{1}{6}(1-u) A \otimes u A-\frac{1}{6}\left(1 \otimes u^{2} A^{2}\right)\right] .
\end{aligned}
$$

In special cases $u=0$ and $u=1, \mathcal{F}_{u}$ reduces to

$$
\mathcal{F}_{0}=e^{-\ln (1+A) \otimes D}, \quad \mathcal{F}_{1}=e^{-D \otimes \ln (1-A)},
$$

respectively.

The corresponding quantum R-matrix is $\mathcal{R}=\tilde{\mathcal{F}}_{u} \mathcal{F}_{u}^{-1}=1 \otimes 1+r+\mathcal{O}\left(\frac{1}{\kappa^{2}}\right)$, where $r=A \otimes D-$ $D \otimes A, \forall u \in \mathbb{R}$.

\section{From realization to star product and twist}

Realizations of noncommutative coordinates $\hat{x}^{\mu}$ can be generally expressed in terms of Heisenberg algebra $H$ (2), generated by $x^{\mu}$ and $p_{\mu}$. If $\hat{x}^{\mu}$ generate a Lie algebra, there exists universal formula for $\hat{x}^{\mu}$, related to Weyl ordering [17]. ${ }^{7}$ Starting from the realization (22) (see for example [18])

$$
\hat{x}^{\mu}=x^{\alpha} \varphi_{\alpha}^{\mu}(p)=\left(x^{\mu}+i a^{\mu}(1-u) D\right)(1-u A)
$$

one can reconstruct the star product using the following method. Recalling the action introduced in section 2 (3) we can explain how the exponent function of the noncommutative coordinates acts on the usual exponential function, via the realization 22 of $\hat{x}$.

$$
e^{i k \cdot \hat{x}} \triangleright e^{i q \cdot x}=e^{i k \cdot x^{\alpha} \varphi_{\alpha}^{\mu}(p)} \triangleright e^{i q \cdot x}=e^{i \mathcal{P}(k, q) \cdot x},
$$

Here we introduced another set of functions, denoted by $\mathcal{P}_{\mu}(k, q)$ which satisfy the following differential equations [19, 20, 21, 22]

$$
\frac{d \mathcal{P}_{\mu}(\lambda k, q)}{d \lambda}=\varphi_{\mu}^{\alpha}(\mathcal{P}(\lambda k, q)) k_{\alpha}
$$

Note that the equation involves the same function $\varphi_{\mu}{ }^{\alpha}(p)$ from the realization of the noncommutative coordinates. The boundary conditions are $\mathcal{P}_{\mu}(0, q)=q_{\mu}$. The solution is

$$
\mathcal{P}_{\mu}(k, q)=\frac{K_{\mu}(k)(1+u(a \cdot q))+(1-(1-u)(a \cdot K(k))) q_{\mu}}{1+u(1-u)(a \cdot K(k))(a \cdot q)},
$$

\footnotetext{
${ }^{7}$ For a more general case, see [22].
} 
where

$$
K_{\mu}(k)=\mathcal{P}_{\mu}(k, 0)=k_{\mu} \frac{e^{a \cdot k}-1}{a \cdot k} \frac{1}{(1-u) e^{a \cdot k}+u} .
$$

The inverse function of $K_{\mu}(k)$, defined as $K_{\mu}\left(K^{-1}(k)\right)=K_{\mu}^{-1}(K(k))=k_{\mu}$, is given/by

$$
K_{\mu}^{-1}(k)=k_{\mu} \frac{1}{a \cdot k} \ln \left(\frac{1+u(a \cdot k)}{1-(1-u) a \cdot k}\right) .
$$

There exists a relation between the function $\mathcal{P}_{\mu}(k, q)$ and introduced before function $\mathcal{D}_{\mu}(k, q)$ as $\mathcal{D}(k, q)=\mathcal{P}\left(K^{-1}(k), q\right)$. Thanks to this, we can rewrite the corresponding star product of exponential functions as

$$
e^{i k \cdot x} \star e^{i q \cdot x}=e^{i K^{-1}(k) \cdot \hat{x}} \triangleright e^{i q \cdot x}=e^{i \mathcal{P}\left(K^{-1}(k), q\right) \cdot x}=e^{i \mathcal{D}(k, q) \cdot x)}
$$

The function $\mathcal{D}_{\mu}(k, q)$ from equation (40) coincides with equation (20).

From the function $\mathcal{D}_{\mu}(k, q)$, the coproduct $\Delta p_{\mu}$ can be recovered using (21) and antipodes $S\left(p_{\mu}\right)$ follow analogously. Alternatively, it is possible to find the coproduct $\Delta p_{\mu}$ as

$$
\Delta p_{\mu}=e^{i K_{\alpha}^{-1}(p) \otimes \mathrm{ad}_{\hat{x}^{\alpha}}}\left(1 \otimes p_{\mu}\right),
$$

From star product and coproduct to twist

From equations (21) and (40) it follows [13, 14, 21]:

$$
\begin{aligned}
e^{i k \cdot x} \star e^{i q \cdot x} & =\lim _{\substack{y \rightarrow x \\
z \rightarrow x}}\left(e^{i x \cdot\left[\mathcal{D}\left(-i \partial^{y},-i \partial^{z}\right)+\partial^{y}+\partial^{z}\right]}\left(e^{i k \cdot y} e^{i q \cdot z}\right)\right) \\
& =m\left[: e^{i\left((1-t) \otimes x^{\alpha}+t x^{\alpha} \otimes 1\right)\left(\Delta-\Delta_{0}\right) p_{\alpha}}:(\triangleright \otimes \triangleright)\left(e^{i k \cdot x} \otimes e^{i q \cdot x}\right)\right], \quad \forall t \in \mathbb{R} .
\end{aligned}
$$

Note that on the right hand side of the above equation the terms multiplied by $t$ cancel each other due to the normal ordering and the multiplication map $m$.

From this identity and equations (4), (19), it follows:

$$
\mathcal{F}^{-1}=: e^{i\left((1-t) \otimes x^{\alpha}+t x^{\alpha} \otimes 1\right)\left(\Delta-\Delta_{0}\right) p_{\alpha}}:+\mathcal{I}_{0}
$$

where $\mathcal{I}_{0} \subset H \otimes H$ is the right ideal of the coordinate algebra $\mathcal{A}$, defined by

$$
m\left[\mathcal{I}_{0}(\triangleright \otimes \triangleright)(\mathcal{A} \otimes \mathcal{A})\right]=0
$$

For the case $u=0$, with $t=0$, twist $\mathcal{F}_{0}^{-1}$ is given by

$$
\mathcal{F}_{0}^{-1}=: e^{A \otimes D}:=e^{\ln (1+A) \otimes D},
$$

while for the case $u=1$, with $t=1$, twist $\mathcal{F}_{1}^{-1}$ is given by

$$
\mathcal{F}_{1}^{-1}=: e^{-D \otimes A}:=e^{D \otimes \ln (1-A)} .
$$

Calculation of $\mathcal{F}^{-1}$ in equation 43 in the form $\mathcal{F}^{-1}=e^{-f}$ for linear realizations of $\hat{x}^{\mu}$ is presented in [21] and for Abelian twists in [13].

Twist in the Hopf algebroid approach 
Deformed phase spaces of Lie algebra type are presented and studied in [15, 23. 26. 27]. Twists in Hopf algebroid approach were studied and constructed in [14, 16, 24, 25].

Generally, in Hopf algebroid approach, the twist is given by

$$
\mathcal{F}^{-1}=e^{-i p_{\alpha} \otimes x^{\alpha}} e^{i K_{\gamma}^{-1}(p) \otimes \hat{x}^{\beta}}+\mathcal{I}_{0}=e^{-i p_{\alpha} \otimes x^{\alpha}} e^{i K_{\gamma}^{-1}(p) \otimes x^{\beta} \varphi_{\beta}^{\gamma}(p)}+\mathcal{I}_{0}
$$

This is a generalization of the result presented in [16]. The full Hopf algebroid analysis will be presented elsewhere.

\section{Concluding remarks}

Inspired by the recent interest in Jordanian deformations, in this paper we focused on the simple generalization of the locally r-symmetric Jordanian twist. By introduction of one real valued parameter $u$ we obtained the family of Jordanian twists which provides interpolation between the original Jordanian twist (for $u=0$ ) and its flipped version (for $u=1$, up to minus sign in the deformation parameter). All of the proposed twists provide the $\kappa$-Minkowski spacetime and have the support in the Poincaré-Weyl or conformal algebras as deformed symmetries of this noncommutative spacetime. Another important issue considered here was the presentation of different methods relating the twist, deformed coproducts with the star-product and the realizations for the noncommutative coordinates.

It is important to note that we can present our results symbolically by the following diagram:

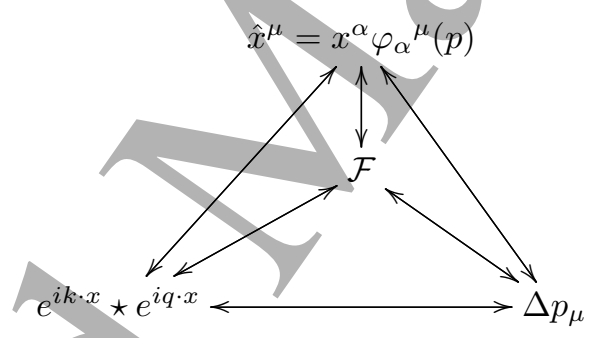

In section 2, starting from the twist (5), we have found deformed Hopf algebra symmetry (7)(10), star products (19), 20) and corresponding realizations for noncommutative coordinates (22). Relation between coproduct $\Delta p_{\mu}$ and star products $e^{i k \cdot x} \star e^{i q \cdot x}$ (19), 20) are given in (21). Noncommutative coordinates $\hat{x}^{\mu}$ are also obtained from the coproduct $\Delta p_{\mu}$ in equation (23) and also from the related star product $e^{i k \cdot x} \star e^{i q \cdot x}=e^{i \mathcal{D}(k, q) \cdot x}$. In section 3 , starting from realizetions of noncommutative coordinates (34), we have constructed corresponding star products (20), (40) and coproducts. Alternatively, coproduct $\Delta p_{\mu}$ is obtained from noncommutative coordinates (41). Also, from the star product and the coproduct in section 2, see also equations (40), (42), inverses of the corresponding twist (43) are obtained. Twists in the Hopf algebroid approach, in terms of momenta and noncommutative coordinates, are given in equation (47). Note that the inverse twist $\mathcal{F}^{-1}$ is not uniquely determined from the star product, coproduct $\Delta p_{\mu}$ and noncommutative coordinates $\hat{x}^{\mu}$ and it is determined up to a right ideal $\mathcal{I}_{0}$.

More general realizations are of the type $\hat{x}^{\mu}=x^{\alpha} \varphi_{\alpha}{ }^{\mu}(p)+\chi^{\mu}(p)$, but we restricted our considerations to the case $\chi^{\mu}(p)=0$ and the above diagram corresponds to $\chi^{\mu}(p)=0$. We point out that all our results are exact. There are few possible extensions of these results, leading to new insights of the deformation quantization. However, they require an extension to the Hopf algebroid 
framework. Drinfeld twists can be lifted to Hopf algebroids [28] from the Hopf algebra, but it is worth to note that not all the twists obtained from the coproduct are the Hopf algebra twists. The issues of the twist reconstruction within Hopf algebroids and the generalization of the other version of symmetrized Jordanian twist presented in [4] will be thoroughly discussed in another paper.

\section{Acknowledgements}

Authors would like to thank A. Borowiec for useful comments. The work by S. M. and D. P. has been fully supported by Croatian Science Foundation under the Project No. IP-2014-09-9582. A. P. acknowledges the funding from the European Union's Horizon 2020 research and innovation programme under the Marie Sklodowska-Curie grant agreement No 660061.

\section{References}

[1] V. G. Drinfeld, "Hopf algebras and the quantum Yang-Baxter equation", Soviet Math. Dokl. $32,254(1985)$

[2] O. Ogievetsky, "Hopf structures on the Borel subalgebra of sl(2)", in: Proceedings of Winter School in Geometry and Physics, Zdikov, January 1993, Supplemento ai Rendiconti del Circolo Matematico di Palermo, Serie II Numero, 37 (1994) 185

[3] V. N. Tolstoy, "Twisted Quantum Deformations of Lorentz and Poincaré algebras", arXiv:0712.3962

V. N. Tolstoy, "Quantum Deformations of Relativistíc Symmetries", arXiv:0704.0081

[4] A. Giaquinto and J. J. Zhang, "Bialgebra actions, twists, and universal deformation formulas", J. Pure Appl. Alg. 128, 133 (1998), arXiv:hep-th/9411140

[5] A. Borowiec, A. Pachoł, "kappa-Minkowski spacetime as the result of Jordanian twist deformation", Phys.Rev.D79:045012,2009, arXiv:0812.0576

[6] D. Kovačević, S. Meljanac, A. Pachoł, R. Štrajn, "Generalized Poincaré algebras, Hopf algebras and kappa-Minkowski spacetime", Physics Letters B 711, 122-127 (2012), arXiv:1202.3305 D. Kovačević, S. Meljanac, "Kappa-Minkowski spacetime, Kappa-Poincaré Hopf algebra and realiza-tions", J.Phys. A: Math. Theor. 45 (2012) 135208, arXiv:1110.0944

[7] J. G. Bu, J. H. Yeê, H. C. Kim, "Differential structure on $\kappa$-Minkowski spacetime realized as module of twisted Weyl algebra", Phys. Lett. B 679, 486 (2009), arXiv:0903.0040

[8] J. Lukierski, A. Nowicki, H. Ruegg, V. N. Tolstoy, "q-deformation of Poincaré algebra" Phys. Lett. B 264, 331 (1991)

J. Lukierski, A. Nowicki, H. Ruegg, "New quantum Poincaré algebra and $\kappa$-deformed field theory", Phys. Lett. B293, 344 (1992)

[9] S. Meljanac, A. Pachoł, D. Pikutić, "Twisted conformal algebra related to $\kappa$-Minkowski space", Phys, Rev. D 92, 105015 (2015), arXiv:1509.02115

[10] A. Pachoł, P. Vitale, " $\kappa$-Minkowski star product in any dimension from symplectic realization", J. Phys. A: Math. Theor. 48 (2015) 445202, arXiv:1507.03523 
[11] A. Borowiec, T. Jurić, S. Meljanac, A. Pachoł, "Central tetrads and quantum spacetimes", Int. J. Geom. Methods Mod. Phys. 13, 1640005 (2016), arXiv:1602.01292

[12] I. Kawaguchi, T. Matsumoto, K. Yoshida, "Jordanian deformations of the $A d S^{5} \times S_{5}$ superstring" JHEP 1404 (2014) 153 arXiv:1401.4855

I. Kawaguchi, T. Matsumoto, K. Yoshida, "A Jordanian deformation of AdS space in type IIB supergravity", JHEP 1406 (2014) 146 arXiv:1402.6147

S. J. van Tongeren, "Yang-Baxter deformations, AdS/CFT, and twist-noncommutatíve gauge theory", Nuclear Physics B 904, 148-175 (2016) arXiv:1506.01023

[13] T. R. Govindarajan, K. S. Gupta, E. Harikumar, S. Meljanac, D. Meljanac, "Twisted Statistics in kappa-Minkowski Spacetime", Phys.Rev.D77:105010,2008, arXiv:0802.1576

[14] T. Jurić, S. Meljanac, R. Štrajn, "Twists, realizations and Hopf algebroid structure of kappadeformed phase space", International Journal of Modern Physics A Vol. 29 (2014) 1450022, arXiv: 1305.3088

T. Jurić, D. Kovačević, S. Meljanac, " $\kappa$-deformed phase space, Hopf algebroid and twisting", SIGMA 10 (2014), 106, 18 pages, arXiv:1402.0397

[15] S. Meljanac, Z. Škoda, M. Stojić, "Lie algebra type noncommutative phase spaces are Hopf algebroids", accepted in Letters in Mathematical Physics, arXiv:1409.8188

[16] S. Meljanac, Z. Škoda, "Hopf algebroid twists for deformation quantization of linear Poisson structures", arXiv:1605.01376

[17] N. Durov, S. Meljanac, A. Samsarov, Z. Škoda, "A universal formula for representing Lie algebra generators as formal power series with coefficients in the Weyl algebra", J.Algebra 309 (2007) 318-359, arXiv:math/0604096

S. Meljanac, S. Krešić-Jurić, T. Martinić, "The Weyl realizations of Lie algebras and left-right duality", J. Math. Phys. 57 (2016), 051704, arXiv:1511.03936

[18] S. Meljanac, S. Krešić-Jurić and M. Stojić, "Covariant realizations of kappa-deformed space", Eur. Phys. J. C 51, 229 (2007), arXiv:hep-th/0702215

S. Meljanac, M. Stojić, "New realizatiơns of Lie algebra kappa-deformed Euclidean space", Eur. Phys. J. C47, 531 (2006), arXiv:hep-th/0605133

[19] S. Meljanac, D. Meljanac, A. Samsarov, M. Stojić, "Lie algebraic deformations of Minkowski space with Poincaré algebra", arXiv:0909.1706

S. Meljanac, D. Meljanac, A. Samsarov, M. Stojić, "Kappa-deformed Snyder spacetime", Mod.Phys.Lett.A25:579-590,2010, arXiv:0912.5087

S. Meljanac, D. Meljanac, A. Samsarov, M. Stojić, "Kappa Snyder deformations of Minkowski spacetime, realizations and Hopf algebra", Phys.Rev.D83:065009,2011, arXiv:1102.1655

[20] S. Meljanac, Z. Škoda, D. Svrtan, "Exponential formulas and Lie algebra type star products", SIGMA 8, 013 (2012), arXiv:1006.0478

[21] T. Jurić, S. Meljanac, D. Pikutić, "Realizations of $\kappa$-Minkowski space, Drinfeld twists and related symmetry algebras", Eur. Phys. J. C (2015) 75:528, arXiv:1506.04955

[22] S. Meljanac, D. Meljanac, F. Mercati, D. Pikutić, "Noncommutative Spaces and Poincaré Symmetry", arXiv:1610.06716 
[23] J. Lukierski, Z. Škoda, M. Woronowicz, "Deformed Covariant Quantum Phase Spaces as Hopf Algebroids", Phys.Lett. B750 (2015) 401-406, arXiv:1507.02612

[24] T. Jurić, S. Meljanac, R. Štrajn, " $\kappa$-Poincaré-Hopf algebra and Hopf algebroid structure of phase space from twist", Physics Letters A 377 (2013), pp. 2472-2476, arXiv:1303.0994

[25] P. Xu, "Quantum groupoids", Commun.Math.Phys. 216 (2001) 539-581, arXiv:math/9905192

[26] J. Lu, "Hopf algebroids and quantum groupoids", arXiv:q-alg/9505024

[27] G. Böhm, K. Szlachányi, "Hopf Algebroid Symmetry of Abstract Frobenius Extensions of Depth 2", Comm. Algebra 32 (11) (2004) 4433 - 4464, arXiv:math/0305136

G. Böhm, "Hopf Algebroids", arXiv:0805.3806

[28] A. Borowiec, A. Pachoł, "Twisted bialgebroids versus bialgebroids from a Drinfeld twist", J. Phys. A: Math. Theor. (2017) accepted for publication arXiv:1603.09280 